\title{
Pursuing Patterns, Puzzles, and Paradoxes
}

By Linda S. Gottfredson

University of Delaware

A true scientist is bored by knowledge; it is the assaulton ignorance that motivates him-the mysteries that previous discoveries have revealed.

Ridley (2000, p. 271)

Although not bored by knowledge, I certainly do love mysteries. It has been my great fortune to earn a living solving them and, greater yet, to pick which puzzles to pursue. I still marvel at the opportunity.

Early in my career I discovered how scientifically important a knotty puzzle or seeming paradox can be. By paradox I mean two well-established findings that seem to contradict each other. Both are accurate because both have been replicated many times. But how can $A$ and not-A simultaneously be true? They cannot, of course, which means that our thinking about them must be flawed in some fundamental respect. Therein lies the opportunity to discover something important.

One advantage of paradoxes is that there is clearly an interesting problem to solve. They also provide special scientific leverage precisely because they pose special challenges. First, they constrain our theorizing about potential explanations. Having a specific contradiction to explain not only provides a well-defined target, but hitting it also requires explanatory discipline. By definition, paradoxes close off familiar paths we would otherwise be predisposed to follow. Second, having to resolve seeming contradictions between two competing bodies of evidence forces one to break set, suspend judgment, and re-examine the deepest conceptual and analytic foundations on which the conflicting conclusions rest. One must look at old evidence with new eyes. Breaking set is perhaps the hardest thing to do. Finally, resolving seeming paradoxes requires throwing a wide net for new ideas and evidence, that is, for embracing the totality of evidence to the extent possible. Seeking relevant knowledge in unfamiliar disciplines increases the odds of serendipitous insights and allows novel empirical patterns to emerge.

Stalking puzzles is only one among other sorts of research I have done, including survey research, policy analysis, secondary analyses of large public datasets, and case studies of professional practice. While solving seeming paradoxes has

been more

demanding and

unpredictable than

other research,

it has also been the most exciting and rewarding. I

describe several puzzles below that instigated long expeditions across various disciplines, which eventuated in explanations for phenomena of general interest. These examples illustrate useful research tactics I discovered in the process, as well as some strikingly counterintuitive lessons in conceptualizing and modeling human behavior.

\section{Four Puzzles and Their Resolution}

The following four puzzles are not all important in themselves, but pursuit of them led eventually to creating theories to account for some previously unexplained human phenomena. By creating a theory I mean mining existing research literatures to build a nomological network of empirical evidence around some phenomenon until a coherent causal pattern appears that can "explain" it.

1. Instigating puzzle: Why do children's vocational aspirations recreate the social class and gender inequalities of their parents' generation long before they ever confront any labor market realities or need to make choices?

Phenomenon explained (in vocational psychology):

1. How various internal and external factors in social and cognitive development successively lead children to circumscribe their perceptions of self and work, and hence their career aspirations, from birth through young adulthood (Gottfredson, 1981, 2002).

2. Instigating puzzle: Why does highest level of education attained best predict who gets good jobs, but intelligence level best predict who performs them well?

Phenomenon explained (in educational sociology): Why the occupational prestige hierarchy represents a hierarchy of increasingly complex and cognitively demanding work tasks, and how the division of labor evolved to distinguish jobs primarily according to overall cognitive complexity of work and only secondarily according to functional field of work (Gottfredson, 1985, 1986).

3. Instigating puzzle: Humans possess a highly general intelligence $(g)$ that has practical value in virtually all life arenas, so how could specific aspects of our evolutionary 
environment have ever selected for such a domaingeneral problem solver?

Phenomenon explained (in evolutionary psychology): How human innovation could have accelerated selection for higher intelligence in our species by disproportionately increasing the risk of accidental death among the least intelligent members of a group while improving the average survival rate overall (Gottfredson, 2007).

4. Instigating puzzle: Why are social class disparities in health and health behavior so pervasive, regular, and linear across such disparate different times, places, diseases, types of health system, and levels of wealth?

Phenomenon explained (in social epidemiology \& health psychology): How inconspicuous cognitive errors in preventing and managing injury and illness cumulate over time, place, and maladies to increase rates of preventable morbidity and mortality disproportionately and linearly across social groups of successively lower average phenotypic (developed) intelligence, such as lower social classes, lower-level occupations, and lesser educated individuals (Gottfredson, 2004; this is the article that won the 2008 George Miller Award from Division 1).

\section{Spotting Contradictions}

Puzzles and contradictions abound. They are not necessarily self-evident, so must often be discovered or discerned. The first three puzzles listed above had not been recognized in their respective disciplines because one half of the contradiction lay isolated in a different discipline: respectively, in (1) sociology, (2) personnel selection psychology, and (3) individual differences psychology. The mystery of the social class-health gradient (4) has occupied health scientists for decades, but that field has for various reasons shut itself off from individual differences psychology and thus a potential resolution.

In the case of puzzle (1), both vocational psychologists and status attainment researchers in sociology were ignoring or dismissing the others' research because there was little if any overlap in their aims, assumptions, methods, and journals. Sociologists tended to be hostile to the notion that individuals differ in skills and interests or that they might voluntarily choose different life paths. Vocational psychologists had recognized the existence of social barriers but not devoted serious attention to them, perhaps because their subject pools usually consisted of college students. The class- and gender-based constriction of vocational preferences from the youngest ages therefore did not draw the attention of either discipline.

In the next two cases (2 and 3), the fact that one set of findings was clearly true was explicitly used by its home discipline to deny that the other set (from another discipline) could possibly be true. I take them in turn. When I was a graduate student in sociology, major thinkers in that field were arguing that intelligence differences had little or no functional importance in real life. One supposed proof rested on the fallacious reasoning that because (a) educational level predicted occupational status level better than did IQ and (b) educational level did not predict on-the-job performance, then (c) intelligence could not possibly predict job performance. In short, one set of findings (from sociology) was used to neutralize the contrary set (from personnel selection psychology), leaving no puzzle for sociologists to explain. Their error had been to conflate what it takes to enter more vs. less prestigious occupations (the sociologists' concern) with what it takes to perform any one of them well once hired (personnel selection psychology's concern). The puzzle is how both relations could hold if employers are behaving rationally when hiring and evaluating workers.

Turning to the other example, puzzle (3), evolutionary psychologists generally argue that all evolutionary adaptations evolved to solve highly specific problems in a species' survival and reproduction. The human brain, they suggest, is like a Swiss Army knife, which has specific blades for specific uses but none of which have value for many uses. Because adaptive problems are specific, the presumption is that the mind must have evolved separate modules to solve them. It cannot be a general problem solver or learning machine, if only because a combinatorial explosion of possible responses would result from the unconstrained processing of information. However, a century of research in individual differences psychology has documented that there is, in fact, a highly general intelligence that confers practical advantages in many areas of life. General intelligence exists, so the puzzle does too. The evolutionary psychologists had erred by rebutting a straw-man general processor, namely, the behaviorist conception of associative learning. The real challenge thus remains-how could a highly general intelligence have evolved?

Contradiction (4), which is the inexplicable generality and linearity of social class-health gradients, shared many features with (1) above. Like sociologists, social epidemiologists tend to favor social-barriers explanations of social inequality. Both have been reluctant to consider the possibility that group differences in occupational and health outcomes, respectively, might arise to any degree from, respectively, average gender and class differences in interests, abilities, and life goals that affect occupational choice and class differences in average intelligence as they affect health self-care. Average group differences in these psychological traits had been replicated many 
times, but social epidemiologists have generally presumed them invalid or inconsequential.

I say this not to criticize the disciplines, past or present, but simply to point out a fact of life. All scientific disciplines work from guiding assumptions, as they must. Their assumptions often differ or conflict, yet remain implicit or taken-for-granted. For example, both vocational psychology (my first adopted field) and status attainment research in sociology (my field of graduate study) seek to understand why different kinds of people end up different kinds of work. But that is where the similarity ends. One arrays occupations horizontally (interest or personality type), and the other vertically (status level). Where one focuses on how individuals differ in interests and abilities, the other treats individuals as psychologically fungible and thus focuses on the external forces blocking their ascent up the social ladder. The first speaks of personal choice, the second of social barriers.

\section{Exploring Across Disciplines}

My experience is that solid, replicable contradictions are more likely to come into view when juxtaposing contrasting bodies of thought and evidence. The contradictions will not be obvious to the disciplinary tourist, but will materialize only after immersion in some part of the foreign discipline or sub-discipline.

Disciplines literally speak different languages, where the same word can mean different things-usually that discipline's favorite part of the metaphorical elephant. So, the word occupation may stand for location in a social hierarchy (sociology), personality type of incumbents (vocational psychology), earnings trajectory (economics), configuration of duties performed (industrial psychology), and so on. All this makes for confusing and fraught cross-disciplinary communication. Doing interdisciplinary research is like moving to a foreign land with a different history, language, and culture. It takes time, exposure, and effort-immersion-to finally "catch on." Only by translating vocational psychology and status attainment into the other could I even begin parsing the first puzzle above. And so it has been with all the others.

One needs to read and think enough in the foreign literature to develop an intuition for its patterns of results, which requires understanding the strengths and limitations of its data. These patterns of results may not be the ones that its own practitioners emphasize, because they come to the data with different aims and assumptions. To understand the empirical evidence, I focus on data and methods. I mostly ignore the authors' own conclusions except as they challenge my own or help me understand how the field reasons.

My initial forays into other disciplines are generally wide- ranging and exploratory, the aim being to acquaint myself with a field's terminology, assumptions, methods, sources of error and doubt, and patterns of results. If already working on a specific puzzle, I look for how the field might contribute to or contradict the nomological network I am accruing around my phenomenon of interest. As a practical matter, this means locating a few top-notch articles or pertinent summaries and then looking at the articles they cite and then, using the Web of Science database, the articles that subsequently cite them. I follow good leads until they either converge or cross the boundaries of relevance often enough to know I have reached the point of diminishing returns-and usually cognitive overload as well.

The fact that some piece of information somehow seems relevant does not necessarily make it useful. One still has to see why it is relevant and how it contributes to resolving the apparent contradiction. A familiar old fact can stare you in the face for months or years before you realize how crucial it is. Here is an example relating the case (2) above.

I spent several years scouring various literatures to find datasets to test the sociological claim that employers hire more intelligent applicants for higher-level jobs, not because they make better workers, but because intelligence is a socially-constructed pretext for justifying the privileges of the privileged classes. A companion claim was that the occupational hierarchy serves no functional purpose but is merely a power hierarchy by which some classes can dominate others. One major theorist was claiming that virtually anyone could do virtually any job if they received the proper training. Much of this was empirical nonsense, but what struck me was that the field took the hierarchy itself for granted. And, except for their hierarchical ordering, the occupations arrayed along it were, for that field, just black boxes that could be filled with anything or nothing. All that really mattered was attached to their surface-prestige, income, and educational requirements.

Now, vocational psychologists had spent many decades grouping occupations according to work activities and developing elaborate procedures for helping clients identify which occupations best matched their interests and capabilities. Military and civilian employers had spent decades analyzing the component duties and aptitude demands of jobs for purposes of worker selection, classification, training, performance evaluation, and setting pay scales. It was these fields' datasets I was merging and analyzing to determine which abilities best predict performance in hundreds of occupations up and down the occupational hierarchy.

My instigating puzzle had forced me to think about why 
employers would hire workers by a qualification that did not actually predict on-the-job performance. After reading some of the microeconomics literature on signaling, I realized that education is a cheap and fallible but reasonably valid way for employers to sort applicants into different occupational bins by average intelligence level. Higher intelligence enhances on-the-job performance to some extent in all jobs (as personnel psychologists were showing), but especially in higher level work because, as I was showing, that work actually is more complex and cognitively demanding.

In proving that the occupational hierarchy is not just a socially constructed excuse for social inequality I myself had taken for granted a crucial phenomenon. Occupations are not discrete boxes of fixed sets of tasks. Rather, they are fuzzy, evolving constellations of tasks that often change depending on the skills and abilities of the individuals performing them. In fact, I had earlier viewed this as a problem in my research; just a nuisance and source of error. How could I classify occupations by aptitude demands if the occupations themselves kept shifting in content? But here, all along, was the mechanism by which the occupational hierarchy could have evolved over human history.

By combining the now-obvious protean nature of occupations with well known facts about human intelligence, I could also explain the surprising finding in sociology that the occupational prestige hierarchy is fundamentally the same worldwide. First, cognitive diversity is a biological fact in all human populations. Moreover, our many distinct abilities tend to line up along a single general dominating dimension, general intelligence or $\mathrm{g}$, which forms the common core of all mental abilities. Second, when work tasks gradually proliferated in kind and number as human groups grew in size and complexity, work tasks would have become segregated on a recurring basis into separate occupations.

Third, the division of labor most likely to survive and reproduce itself over generations would be one in which its various sectors have reliable inflows of workers who have the requisite combinations of aptitudes for learning and carrying out that work. A major recurring biological constraint on such flows would be the genetically-influenced structure of human differences in ability. As noted earlier, those differences are organized primarily according to general intelligence level and only secondarily according to profile differences in verbal vs. spatial, and so on, meaning the division of labor would have to be too. And so it is (Gottfredson, 1986).

\section{Writing to Impose Explanatory Rigor}

Broad exploration is fun, interesting, and relatively easy.
It can generate lots of ideas. The hard work comes in winnowing, organizing, and testing them to develop a plausible explanation. Good explanations are tight databased arguments, not compendia of facts or fanciful speculation. In my experience, only writing provides the necessary intellectual discipline to develop a rigorous, compelling argument. I therefore start writing when I glimpse an answer to the paradox. I do not stop exploring, but focus it more tightly.

To be clear, all four publications began as manuscripts that had been either accepted or invited for publication. At some point I had to start writing and eventually produce a publishable manuscript. In no case, however, did I know exactly what I would say. I knew only that I had a puzzle I wanted to work through.

In the first case, (1), I had temporarily withdrawn a little 8-page paper from publication in order to add a missing paragraph. My struggle to get it right ended up 6 months later as a monograph. My short conceptual piece argued that vocational choice proceeds by children rejecting the least acceptable options for someone like themselves, not by identifying the best. Even before adolescents start consciously thinking about vocational options, they have already ruled out whole swaths of the occupational world that might actually fit their abilities, interests, and life goals, but which they are not likely to revisit unless prompted to do so. My subsequent effort to explain how this process unfolds and provide evidence for it forced me to look back into my notes, rummage again through my accumulating piles of books and articles, and venture out into new corners of the literature in order to answer more specific questions and test new hypotheses. "What does the literature say about preschoolers' conceptions of gender differences?" "Do the bases of rejection shift as children become cognitively able to recognize more abstract dimensions of our shared social order (gender, class, ability, personality, and so on)?" "At what age do children's perceptions of the social order mirror those of adults?" I spent months alternating between writing and returning to the vocational, developmental, and sociological literatures for specific sorts of information.

In case (3), I started writing when I had the insight that the relatively sudden evolution of Homo sapiens sapiens' general intelligence could have been driven by the proliferation of evolutionarily novel, low-probability causes of death created as side-effects of human innovation. Human groups organize to protect themselves against the most obvious threats to survival-war, starvation, weather, and predation-not against ones they think random or "accidental." No specific modules could have evolved to protect against each of the many low-probability causes of death that proliferated with technological advance-such 
as clothes catching fire, drowning while fishing off piers or boats, accidentally nicking oneself with a poisoned dart, or being gored by domesticated animals. These are the same evolutionarily novel hazards that still cause so much unintentional injury and death today: piercing, cuts, falls, crushing, burns, scalds, poisons, dog bites, and so on.

Unintentional injury is the major cause of death from toddlerhood through early middle age in both developed and developing countries. This is what had led me to look at the industrial accident literature when I was working on the puzzle of the social class-health gradient, (4). The accident literature described what now seemed obvious in hindsight-hazards are ubiquitous, the key question is not what causes accidents but what prevents them, and prevention is a quintessentially cognitive process. The accident prevention process could explain how the hazards created by human innovation might put less intelligent members of a group at slightly higher risk of death during their reproductive years. Evolution works with such tiny differences in risk.

I consulted anthropological accounts of hunter-gatherer groups to test my deadly-innovations hypothesis. I found that accidental death was indeed a major cause of death in pre-literate societies, but the evidence also showed that the deadly innovations I had in mind were far too recent to account for the evolution of high human intelligence. For instance, pre-contact Ache in the Twentieth Century used fire, had digging and cutting implements, and hunted with bows and arrows, but few of them were fatally injured by those implements (Hill \& Hurtado, 1996). Far more had died from stepping on poisonous snakes. In any case, bows and arrows are a relatively recent human innovation.

But something had to have selected for we human's highly general ability to learn and reason, so I began searching for evidence and speculation about the earliest Homo sapiens and their environments. What about more basic hunting, gathering, and cooking technologies? A passage in The Promethean Fire (Lumsden \& Wilson, 1983) made me realize, with a jolt, that I had falsely equated human innovation with physical technology. Innovation is more basic than that. It is simply having a mind's eye: that is, being able to imagine something beyond what we are seeing, feeling, tasting or otherwise experiencing in the present moment; to imagine times, events, objects, beings, and circumstances that do not exist now and may never. To become tool makers and technologists we first had to become imaginators, to see beyond the concrete here and now. Recognizing hazards as potential threats to well-being is an act of imagination.

With that insight, I re-examined the hunter-gatherer reports. Death by stepping on snakes occurred while hunters were looking into the forest canopy for monkeys, their attention diverted upward by being able to kill them at a distance owing to their bow and arrow technology. Even primitive projectiles would pose the same hazard. Innovations that divert attention, create physical forces that exceed normal human tolerances, or expose individuals to more hazards or less familiar ones increase the need for prevention, and hence effective reasoning and "foresight."

The foregoing experiences illustrate how the writing process provides discipline and focus, and is thus integral to conducting this kind of research. Writing is part of the expedition, not a report on it, because it helps expose flaws in logic, gaps in the evidence, and missing links in the chain or argument: "What do I really mean here? This doesn't follow or feel quite right. How can I account for X? Might there be data on that?"

Writing can also impose blinders, however. Tight focus in the last thing one needs if heading down the wrong path. The sense of encountering a writing barrier usually signaled my having bumped up against some conceptual problem. Thinking is really hard work, as I tell my students, but just doggedly pushing ahead can get you deeper into a deadend corner, especially if you are fatigued. Strategic timeouts may help one return with fresh eyes. Everyone finds their own style, but I have discovered that it helps to let my mind wander on a particularly vexing problem when I am relaxed or carrying out tasks not requiring much cognitive effort. Ideas can play and rearrange themselves, letting crucial similarities, distinctions, or bits of information pop into the foreground.

\section{Reconceptualizing Models and Methods}

These sorts of cross-disciplinary research expeditions could be called theoretical syntheses, inductive pattern-analysis, or perhaps meta-research. My expeditions are usually unplanned and unanticipated, their conduct messy, the evidentiary guide-posts necessarily error-ridden, the end-point a surprise, and the publication lengthy. But if successful, the resulting explanation will seem transparent and the evidence for it obvious in hindsight. It is a research method without set procedures. It does not rely on statistical tests or mathematical modeling, yet has provided unanticipated lessons about both.

I like statistics and mathematical modeling, and read about them for pleasure when I can. But when mechanically applied as accepted methodology, they are apt to obscure rather than illuminate the structure of evidence. Their mechanical application short-circuits hard thinking about what the measured variables mean conceptually, why those constructs would or would not be causally related, how they are distributed in the population, and whether statistical significance tells us anything useful about them. 
This became clear to me in graduate school. The recent methodological advances that established status attainment research as avant garde in stratification research-regression analysis and path analysisseemed likely to ensconce rather than expose consensual misconceptions. The new statistical modeling was useful for probing large datasets, but the precision of its parameter estimates conferred only the illusion of validity. Its users were rechristening conceptual questions as statistical problems, such as multicollinearity and the omitted variable problem. I remain skeptical of all statistical and mathematical modeling procedures that pre-process evidence while leaving little trace of its original structure. Hence my preference for starting out close to the data by scrutinizing frequency distributions, means, standard deviations, how variables are actually measured, degree of measurement error, zero-order correlations, and sample composition.

For me, the two most deceptive words in social science are controlled for, as in "We controlled for $X$ to see what influence $Y$ has on $Z$," because there is usually little empirical basis for presuming that particular causal model-and often good reason to doubt it. For example, social scientists routinely rule out empirically plausible explanations for disparities in success and well-being by controlling away valid variance as mere "confounding." This reflex to "statistically control for" has long stalled progress in pinning down causal relations in human behavior. The question, then, is how to make progress despite the inferential ambiguities of non-experimental data obtained from samples that are not genetically informative either.

As already described, it is useful to triangulate many sorts of evidence. Another is to make non-obvious predictions that other proposed explanations would not. I have been able to do that to some extent by calling upon two heuristics that allow me to apply one discipline's body of evidence to another discipline's research problems.

In the case of puzzle (4), I analyze chronic diseases such as diabetes, and health self-care in general, as having a job. Chronic diseases mirror paid jobs in requiring training, having regular duties to perform, being cognitively complex, and requiring self-direction and independent decision making. Quick consultation with the employee selection literature reveals that these task demands put a premium on proficient learning and reasoning, and hence on higher intelligence. The job analysis literature then illuminates which particular aspects of these jobs are more complex and thereby create special risks-or cognitive barriers-in health self-care. Comparing chronic diseases to paid work also shows that the former are more demanding in crucial ways, for instance, in allowing no evenings or weekends off, and no retirement. Moreover, the heuristic makes immediate sense to health care practitioners and patients, and implications for patient education, supervision, and job simplification follow directly from it.

The second heuristic was supplied by a colleague, fellow sociologist Robert Gordon, in an article on "life as a test" (1997). It has had a most profound effect on my thinking, both conceptual and statistical. He describes the ways in which routine daily activities do and do not resemble items on psychometric tests of intelligence, including how standardized they are and the degree to which they call upon $g$, or general intelligence. The most important point for me was that single IQ test items never measure intelligence very well. Indeed, they are individually quite poor measures of it. Their power comes with aggregation, whereby their common variance cumulates and their errors cancel each other out. Applying the SpearmanBrown Prophecy Formula for test reliability, we can calculate how many items are required to produce a test that measures virtually nothing but $g$, depending on how $g$-loaded its items are. If items are only weakly $g$-loaded, we just need more of them.

This life-as-a-test heuristic was central in resolving puzzles (3) and (4). In the case of the evolution of human intelligence, (3), each hazard can be conceived as a weak test item. The risk of accidental death would be miniscule for each. Moreover, each would be only lightly $g$-loaded, the greater part of the variance in injury and death being random ("accidental"). Yet, when cumulating tiny differences in $g$-related risk over myriad hazards, many individuals, and many generations, the odds will aggregate against less intelligent members of the species. No selection factor operating over evolutionary time would be noticeable within a generation, which means, counter-intuitively, that any process obvious to the naked eye would be implausible as a selection factor.

Turning to the puzzle of the social class-health gradient, (4), we see the same principle at work in the daily prevention and management of injury and illness. Maintaining good health requires consistent effort on a daily basis to prevent illness and injury and to limit the damage they cause. Preventive efforts are likely to be less frequent, less consistent, and less effective among individuals who cannot conceive the risks of failing to expend effort that has no obvious payoff. Psychometric principles also tell us which kinds of outcome criteria will best capture the consequences of poor or inconsistent performance. Specifically, they will include outcomes that cumulate over more items (time, events, behaviors), are more reliably measured, depend more heavily on patients exercising their own independent judgment, and instances where 
patients differ more widely in intelligence. That is, the life-as-a-test heuristic suggests novel predictions about when and where disparities in health and health behavior will be greatest.

Both heuristics encourage scrutiny of the distribution of the external task demands that people face in daily affairs. Psychology has many instruments for ascertaining distributions of abilities, but few for the distribution demands that social and physical environments make of us. Both heuristics also caused me to question one of our most basic methodological assumptions, namely, that bigger effects (larger correlations, standardized mean differences, etc.) are necessarily better, more informative, and more important than small ones, and, conversely, that inconspicuous effects are obviously unimportant. Evolutionary logic and the Spearman Brown Formula suggest, however, that consistency of effect may outweigh size of immediate effect when reliably small effects have a chance to cumulate and compound over long periods of time and large populations. This means that effect sizes cannot be directly compared when measures represent different degrees of aggregation, as would be the case for gradepoint average vs. single achievement tests, or an A1c laboratory result vs. a one-shot measure of blood sugar.

Both heuristics now have me pondering how to represent and analyze the topography of effect sizes across different health tasks and groups of individuals. Their pattern could help explain the mystery of the classhealth gradient. Such analyses would require gathering the same parameters, such as regression slopes, for different combinations of predictors and outcomes in the same study and from different studies as well.

If my intelligence-based explanation of the class-health gradient is correct, we should find systematic, predictable differences in gradients. For strictly statistical reasons, gradients should be steeper (slopes larger) when predictors and criteria are more reliably measured, there is less sampling error, and there is less restriction in range in the sample. For substantive reasons, I would expect gradients to be steeper when health tasks are more complex, predictors correlate more highly with patient intelligence, there is greater need or opportunity for patients to exercise independent judgment, and the health behavior or outcome measure is more cumulative in nature. Here is a case where meta-research may intersect meta-analysis.

In summary, there is no recipe for doing good science. I have tried to describe some strategies, however, that I have found useful. Foremost among them are to read widely on phenomena that intrigue me, pin down why perspectives on them differ and what each has to offer, try to locate the essential core of available evidence, not put much stock in any single study or piece of evidence, watch for recurring patterns in the most reliable data, think through likely counterarguments, and look hard for holes in my evidence and logic. Most important, be open to having your basic presumptions shattered. As with good mystery books, the endings are often not what you expect.

\section{References}

Gordon, R. A. (1997). Everyday life as an intelligence test: Effects of intelligence and intelligence context. Intelligence, 24(1), 203-320.

Gottfredson, L. S. (1981). Circumscription and compromise: A developmental theory of occupational aspirations. Journal of Counseling Psychology (Monograph), 28 (6), 545-579.

Gottfredson, L. S. (1985). Education as a valid but fallible signal of worker quality: Reorienting an old debate about the functional basis of the occupational hierarchy. In A. C. Kerchoff (Eds.) Research in Sociology of Education and Socialization, Vol. 5 (pp. 119-165). Greenwich, CT: JAI Press.

Gottfredson, L. S. (1986). Occupational aptitude patterns map: Development and implications for a theory of job aptitude requirements (Monograph). Journal of Vocational Behavior, 29, 254-291.

Gottfredson, L. S. (1997). Why g matters: The complexity of everyday life. Intelligence, 24(1), 79-132.

Gottfredson, L. S. (2002). Gottfredson's theory of circumscription, compromise, and self-creation. Pages 85-148 in D. Brown (Ed.), Career choice and development (4th ed.). San Francisco: Jossey-Bass.

Gottfredson, L. S. (2004). Intelligence: Is it the epidemiologists' elusive "fundamental cause" of social class inequalities in health? Journal of Personality and Social Psychology, 86(1), 174-199.

Gottfredson, L. S. (2007). Innovation, fatal accidents, and the evolution of general intelligence. In M. J. Roberts (Ed.), Integrating the mind: Domain general versus domain specific processes in higher cognition (pp. 387-425). Hove, UK: Psychology Press.

Hill, K., \& Hurtado, A. M. (1996). Ache life history: The ecology and demography of a foraging people. New York: Aldine de Gruyter.

Lumsden, C. J., \& Wilson, E. O. (1983). Promethean fire: Reflections on the origins of the mind. Cambridge, MA: Harvard University Press.

Ridley, M. (2000). Genome: The autobiography of a species in 23 chapters. New York: Harper Perennial. 